\title{
Structured light excitation of toroidal dipoles in dielectric nanodisks
}

\author{
Reza Masoudian Saadabad $\odot,{ }^{1}$ Marcus Cai $\odot,{ }^{2}$ Fu Deng, ${ }^{3}$ Lei Xu,,${ }^{4, *}$ and Andrey E. Miroshnichenko ${ }^{1, \dagger}$ \\ ${ }^{1}$ School of Engineering and Information Technology, University of New South Wales, Canberra, ACT 2600, Australia \\ ${ }^{2}$ Department of Electronic Materials Engineering, Research School of Physics, \\ The Australian National University, Canberra, ACT 2601, Australia \\ ${ }^{3}$ Guangdong Provincial Key Laboratory of Nanophotonic Functional Materials and Devices, School of Information and Optoelectronic \\ Science and Engineering, South China Normal University, Guangzhou 510006, China \\ ${ }^{4}$ Advanced Optics \& Photonics Laboratory, Department of Engineering, School of Science \& Technology, Nottingham Trent University, \\ Nottingham NG11 8NS, United Kingdom
}

(Received 24 May 2021; revised 20 September 2021; accepted 23 September 2021; published xxxxxxxxxx)

Conventional electromagnetic multipoles can be completed by complementary sources of toroidal moments, opening the door to the engineering of a unique nanophotonic device group. The main contribution of this study is comparing different light sources for enhancing the toroidal dipole response in a given system. We theoretically study the toroidal dipole excitation in an individual dielectric nanodisk by structured light illumination, including the tightly focused radially polarized beam and the focused doughnut pulse. The toroidal dipole and anapole can be excited by the interplay of the radial and longitudinal components of the incident light. As opposed to the plane wave illumination, the tightly focused radially polarized light can excite a near-ideal toroidal dipole while the contributions of the Cartesian electric dipole and other modes are significantly suppressed. We also show that the focused doughnut pulse is a promising tool for exciting a resonant toroidal response in nanophotonic systems. Furthermore, it is demonstrated that toroidal-driven field confinement leads to an enhancement of energy concentration inside the nanodisk that can potentially increase light harvesting and boost both linear and nonlinear light-matter interactions.

DOI: 10.1103/PhysRevB.00.005400

\section{INTRODUCTION}

Nanoparticles have been known as an efficient tool to control light-matter interaction after a seminal publication by the German physicist Gustav Mie in 1908 [1]. The paper explained why colloidal solutions with different gold nanoparticles indicate different colors. Over the past decades, they have attracted massive interest due to a wide range of applications in optoelectronic devices such as solar cells and light sources [2]. Compared to the plasmonic counterpart, dielectric nanoparticles are up and coming candidates for the control of light-matter interaction due to the support of optically induced magnetic response that provides an extra degree of freedom to the system [3].

Expansion of an induced current density inside a nanoparticle into a set of pointlike sources, called multipoles, is an efficient tool for analyzing its interaction with light. The expansion commonly consists of Cartesian electric and magnetic moments. Still, in the absence of magnetic charges, the multipole paradigm can be completed by complementary electromagnetic sources called toroidal moments [4]. The lowest-order moment family member is the toroidal dipole moment corresponding to the poloidal currents that flow on a sphere along its meridians. In the interaction between a

\footnotetext{
*lei.xu@ntu.ac.uk

†andrey.miroshnichenko@unsw.edu.au
}

dielectric nanoparticle and an incident electromagnetic field, the toroidal dipole moment represents the contribution of poloidal current to the far-field scattering. Although the electromagnetic wave can induce a toroidal dipole moment, its observation is often tricky as they are usually masked by the contribution of the conventional electric and magnetic moments. The spatial scale of the toroidal dipole in the multipole expansion is $\sim\left(\frac{R}{\lambda}\right)^{3}$ where $\lambda$ refers to the wavelength of the incident wave and $R$ is the characteristic length of the particle; therefore it is observable when the particle size is not small compared to the wavelength [5]. Excitation of the toroidal dipole in dielectric nanoparticles can provide the condition of the radiationless anapole state coming from the out of phase interference of the electric dipole moment with the toroidal dipole moment $[3,6]$. There are also superdipole states $[7,8]$ formed by the constructive overlap of those two moments, and the magnetic anapole [9], referring to the destructive overlap of a magnetic dipole moment and its corresponding toroidal moment. Electric field confinement in a dielectric nanoparticle via anapole excitation has been recently demonstrated to enhance light harvesting [10] that is crucial for photovoltaic systems [11,12] and photodetectors [13-15]. More recently, boosting toroidal response in a metasurface of $\mathrm{Si}$ nanoparticles has led to a 30-fold increase in light absorption [16]. Furthermore, identifying higher-order toroidal multipoles is essential for understanding light interaction with dielectric particles and nanophotonics applications [8]. The study of the resonant excitation of toroidal modes is thus of much interest. 
Tailoring of light in full degrees of freedom to create desired spatiotemporal beams and pulses is referred to as structured light. It is achieved by reshaping the initial plane wave to another optical field configuration with the desired polarization, phase, and amplitude using the various phase or amplitude control approaches such as spatial light modulations, metasurfaces, and variable transmission filters. As opposed to various methods available to generate structured light, their detection is usually carried out by qualitative analysis. For instance, the space-varying polarization of a vector beam means that its intensity pattern will change after passing through a polarizer and this change will be dependent on the polarizer axis [17]. Structured lights have diverse applications from fundamental science to practical applications in optical communication and industrial manufacturing [18-21]. They enable one to excite dark modes of nanostructures that cannot be accessed by light with linear polarization [22,23]. In optical communication, their spatial modes serve as an additional degree of freedom to increase the capacity of information [24,25], and more recently, they have been suggested for encoding information [26].

In this research, we compare different light sources for enhancing the toroidal dipole response in a given system. There are some studies $[27,28]$ investigating the structured light excitation of toroidal dipoles in dielectric nanoparticles, but more work needs to be done on quantitatively comparing the toroidal dipole response under different illuminations. We study the toroidal excitation in an individual dielectric nanodisk via its interaction with the plane wave and more complex waveforms, including the tightly focused radially polarized beam [29] and the focused doughnut pulse $[5,30]$. Despite extensive research on toroidal excitations by the plane wave [31-35], structured light is of more interest due to its potential for exciting a dominant toroidal response in the geometrically simple nanosystems. It also enables new mechanisms and applications emerging in nanophotonics [26,3639]. We show that structured light can strongly interact with the dielectric nanodisk due to the contributions of radial and longitudinal components of the electric field supporting the oscillating displacement currents along the nanodisk's meridians. An excellent toroidal dipole can be excited while the Cartesian electric dipole is eliminated, leading to field enhancement and energy concentration within the particle. Such a toroidal dipole excitation may be used for absorption enhancement and boosting nonlinear light-matter interaction in all-dielectric nanophotonics. It is worth mentioning that all Cartesian multipoles have their toroidal counterparts, such as the second-order toroidal correction of an electric dipole and the toroidal magnetic dipole [8], that go beyond the scope of our study, but can be subject to future research.

\section{TOROIDAL DIPOLE IN THE MULTIPOLE EXPANSION}

In the absence of free charges, an electrodynamic system can be described by a current density $\boldsymbol{J}(\boldsymbol{r}, t)$. Based on the Helmholtz theorem [40], the current density inside a particle can be written in terms of scalar potential $\varphi$ and vector potential $\mathbf{A}$,

$$
\boldsymbol{J}(\boldsymbol{r}, t)=\nabla \varphi+\nabla \times \mathbf{A}=\boldsymbol{J}_{\|}+\boldsymbol{J}_{\perp},
$$
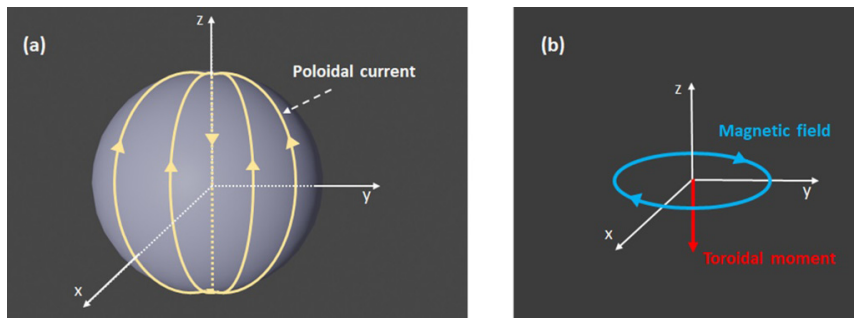

FIG. 1. (a) Poloidal currents and (b) their corresponding magnetic field distribution that is associated with the excitation of the toroidal moment.

where $\boldsymbol{J}_{\perp}$ is the solenoidal component, i.e., $\boldsymbol{\nabla} \cdot \boldsymbol{J}_{\perp}=0$, and $\boldsymbol{J}_{\|}$is the longitudinal component of the current density. The bound charge conservation law $\left(\boldsymbol{\nabla} \cdot \boldsymbol{J}=-\frac{\partial \rho}{\partial t}\right)$ is therefore satisfied by $\boldsymbol{J}_{\|}$. The expansion of $\boldsymbol{J}_{\|}$is characterized by the multipole moments depending on bound charge density, while $\boldsymbol{J}_{\perp}$ is independent of charge moments and determined by two other families of moments. Assume that the neighborhood of the center point $\boldsymbol{r}=0$ represents a small sphere; then one moment is characterized by the toroidal currents that flow along the sphere's parallels. The second is characterized by the poloidal currents flowing over the surface along the meridians and continuing with an internal flow from the north pole to the south pole [Fig. 1(a)]. Excitation of toroidal electric moment $T[4,41]$ is attributed to a confined magnetic field created by the poloidal currents as illustrated in Fig. 1(b).

There are two representations for analyzing the electromagnetic properties of dielectric nanoparticles. One is the multipole decomposition of electromagnetic fields in terms of the spherical harmonic coefficients. The total scattering cross section studies the radiation properties as a series of the spherical multipole scattering coefficients:

$$
\boldsymbol{C}_{\mathrm{sca}}^{\mathrm{sph}}=\frac{\pi}{\boldsymbol{k}^{2}} \sum_{\boldsymbol{l}=1}^{\infty} \sum_{\boldsymbol{m}=-1}^{\boldsymbol{l}}(2 \boldsymbol{l}+1)\left[\left|\boldsymbol{a}_{\boldsymbol{E}}(\boldsymbol{l}, \boldsymbol{m})\right|^{2}+\left|\boldsymbol{a}_{\boldsymbol{M}}(\boldsymbol{l}, \boldsymbol{m})\right|^{2}\right],
$$

where $a_{E}(l, m)$ is the spherical electric multipole scattering coefficient, and $a_{M}(l, m)$ represents the spherical magnetic multipole scattering coefficients [32,42].

The second representation is the multipole decomposition of the Cartesian components of current density inside the particle. Toroidal multipoles appear via the induced current density expansion inside the particle [32]:

$$
\boldsymbol{J}(\boldsymbol{r}, t)=\sum_{l=0} \frac{(-1)^{l}}{l !} B_{i \cdots k}^{(l)} \partial_{i} \cdots \partial_{k} \delta(\boldsymbol{r}),
$$

where $B_{i \ldots k}^{(l)}$ (a tensor of rank $l$ ) represents the multipole moments, and $\boldsymbol{r}$ is the vector from the center point of the coordinates to an arbitrary point of the current distribution area. When the nanoparticle is illuminated by an electromagnetic wave, the electric dipole and toroidal dipole moments in the Cartesian representation ( $\boldsymbol{P}_{\text {car }}$ and $\left.\boldsymbol{T}_{\text {car }}\right)$ are expressed as [37]

$$
\boldsymbol{P}_{\mathrm{car}}=\frac{i}{\omega} \int \boldsymbol{J} d^{3} r, \quad \boldsymbol{T}_{\mathrm{car}}=\frac{1}{10 c} \int d^{3} r\left[\boldsymbol{r}(\boldsymbol{r} \cdot \boldsymbol{J})-2 r^{2} \boldsymbol{J}\right],
$$


where $c$ is the speed of light, $\omega$ is the angular frequency, and $\boldsymbol{J}$ is obtained from

$$
\boldsymbol{J}=-i \omega \varepsilon_{0}\left[n^{2}-1\right] \boldsymbol{E},
$$

where $\varepsilon_{0}$ is the electric permittivity of the vacuum, $n$ shows the refractive index of the particle, and $\boldsymbol{E}$ is the electric field inside the particle calculated by solving Maxwell's equations using the finite element method (FEM). The contribution of the electric and toroidal moments to the total scattered field is [32]

$$
\boldsymbol{E}_{\mathrm{sca}} \sim \frac{k^{2}}{4 \pi \varepsilon_{0}}\left(\boldsymbol{n} \times \boldsymbol{P}_{\mathrm{car}} \times \boldsymbol{n}+i k \boldsymbol{n} \times \boldsymbol{T}_{\mathrm{car}} \times \boldsymbol{n}\right) .
$$

The relation shows that the electromagnetic radiation of a toroidal dipole repeats the pattern of an electric dipole moment scaled by a multiplication $i k$ where $k$ refers to angular wave number. In the far field, as a result, their radiations are not distinguishable. Therefore, the electric dipole and the toroidal dipole cannot be separated when the spherical multipole decomposition of electromagnetic fields is used.

It can be seen from Eq. (6) that far-field radiation becomes zero when the contributions of the electric dipole and the toroidal dipole moments in the scattering are out of phase:

$$
\boldsymbol{P}_{\mathrm{car}}=-i k \boldsymbol{T}_{\mathrm{car}} .
$$

This is the necessary condition for the excitation of a radiationless anapole state. The scattering intensity of the electric and toroidal multipoles are also calculated by [37]

$$
I_{\mathrm{sca}} \sim \frac{2 \omega^{4}}{3 c^{3}}\left|\boldsymbol{P}_{\mathrm{car}}\right|^{2}+\frac{2 \omega^{6}}{3 c^{5}}\left|\boldsymbol{T}_{\mathrm{car}}\right|^{2}+\frac{4 \omega^{5}}{3 c^{4}} \operatorname{Im}\left(\boldsymbol{P}_{\mathrm{car}}^{\dagger} \cdot \boldsymbol{T}_{\mathrm{car}}\right),
$$

where the last term points to the constructive or destructive interference between the electric and toroidal dipoles.

\section{EXCITATION OF TOROIDAL DIPOLE}

A resonant toroidal dipole can be excited through two approaches: One is engineering dielectric nanostructures that have toroid geometry, and the second is the design of properly polarized laser pulses $[27,28,31]$. The latter is more promising for exciting substantial toroidal response in the geometrically simple systems with nontoroidal configuration. It arises from the fact that linearly polarized light has an electromagnetic field preserved by the space-inversion symmetry. Therefore, careful engineering of subwavelength structures that can break the space-inversion symmetry is required for inducing dominant toroidal response [27,43]. This section will discuss toroidal dipole excitations in an individual highindex dielectric nanodisk by a plane wave and structured light, including the tightly focused radially polarized beam and the focused doughnut pulse. The refractive index of the nanodisk is $n=3.5$, and the surrounding medium is air. To study the interaction of the pulse with the dielectric nanodisk, we considered an idealized nondispersive nanodisk. Interactions between light and the nanodisk were simulated through solving Maxwell's equations by the finite element method on COMSOL MULTIPHYSICS. (a)
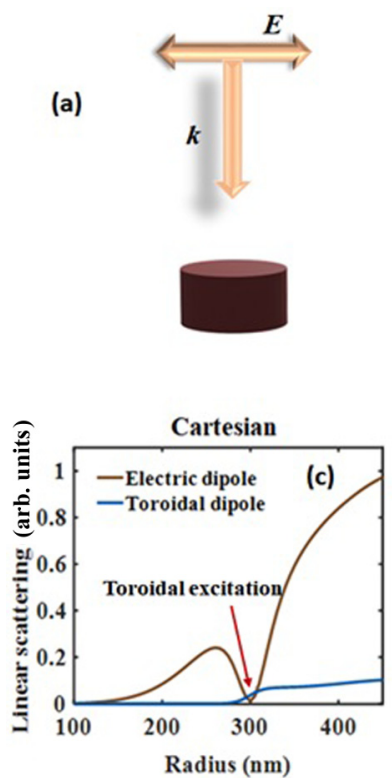
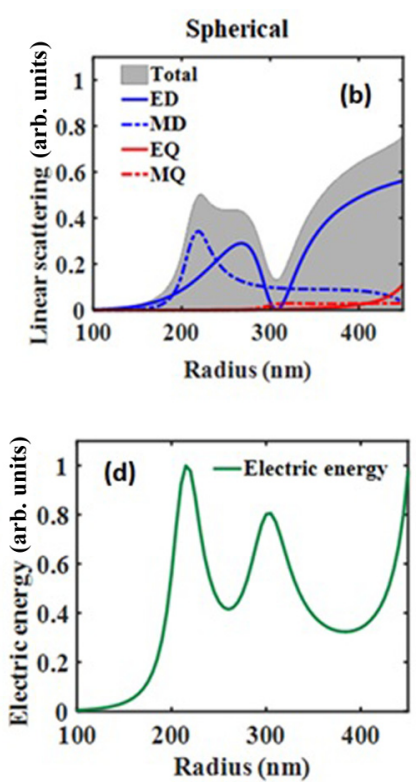

FIG. 2. (a) Configuration of the toroidal dipole excitation where an electromagnetic plane wave illuminates a dielectric nanodisk. (b) Contributions of multipoles up to quadrupole order in the spherical representation. (c) Cartesian electric and toroidal dipoles. The red arrow indicates the toroidal dipole excitation when $P_{\text {car }}=0$ and $P_{\text {sph }} \neq 0$. (d) Electric energy within the nanodisk as a function of radius. The toroidal dipole led to a concentration of electric energy inside the nanodisk of $300 \mathrm{~nm}$.

\section{A. Plane wave}

Among various solutions of Maxwell's equations explaining the electromagnetic radiation behavior, the plane wave of infinite energy $[\boldsymbol{E} \& \boldsymbol{H} \sim \exp (i k \boldsymbol{r}-\omega t)]$ is the well-known solution. As the excitation of a toroidal dipole by plane wave illumination in a nanoparticle possessing spherical geometry is generally accompanied by a strong contribution of higher multipoles [32], we investigated the excitation of the toroidal dipole in a nanodisk. Configuration of the excitation is illustrated in Fig. 2(a), where an electromagnetic plane wave $(\lambda=$ $1550 \mathrm{~nm}$ ) illuminates the nanodisk of radius $R$ and height $300 \mathrm{~nm}$. We first analyzed the multipolar decomposition up to quadrupole in order to understand the characteristics of the optical response. Spherical representation of the multipole expansion up to quadrupole order as a function of the nanodisk radius is presented in Fig. 2(b). The scattering spectrum is dominated by two conventional electric and magnetic dipoles over the full radius range. Furthermore, for the radii larger than $300 \mathrm{~nm}$, higher modes, including electric and magnetic quadrupoles, start to contribute to the scattering. There is also a dip in the total scattering around $310 \mathrm{~nm}$ corresponding to an anapole excitation. To confirm this, we calculated the electrical dipole contribution in the Cartesian representation and illustrated the result in Fig. 2(c). It splits the spherical electric dipole into the Cartesian electric and toroidal dipoles. As expected, the contribution of the dipole toroid becomes more significant for larger particles due to the increase of the size to wavelength ratio $\frac{R}{\lambda}$. Destructive interference between the Cartesian electric and toroidal dipoles, then, led to the excitation of the anapole around $310 \mathrm{~nm}$. At this state the 
spherical electric dipole moment (that refers to the scattered field) is zero, $\boldsymbol{P}_{\text {sph }} \sim \boldsymbol{P}_{\text {car }}+i k \boldsymbol{T}_{\text {car }}=0$, while the Cartesian electric dipole moment (that indicates the induced charge polarization inside the nanodisk) is nonzero; i.e., $\boldsymbol{P}_{\text {car }} \neq 0$. This phenomenon led to a suppression in the total scattering manifesting itself as a dip in the spectrum of Fig. 2(b). Here we are interested in the opposite phenomenon: While the induced electric dipole moment inside the nanodisk is absent, a contribution to the far field exists; i.e., $\boldsymbol{P}_{\text {car }}=0$ and $\boldsymbol{P}_{\text {sph }} \neq 0$. This is related to the toroidal dipole excitation at the radius of $300 \mathrm{~nm}$ marked by the red arrow in Fig. 2(c). The electric dipole is zero, but the toroidal dipole contributes to the far-field scattering. Nevertheless, the anapole and the toroidal excitation are not strong and are easily masked by the other contributing modes. As a result, we can see the magnetic dipole in conjunction with quadrupole modes account for $75 \%$ of the multipole scattering at the resonant toroidal excitation.

Tight field confinement associated with toroidal modes may enable one to concentrate energy in the subwavelength volumes. Therefore, it is anticipated that toroidal dipole excitations result in an enhancement of energy concentration inside the nanodisk. To investigate this, we calculated electric field energy within the nanodisk $\left(W=\frac{1}{2} \int|\boldsymbol{E}|^{2} d r\right)$ as a function of its radius in Fig. 2(d). There is a prominent peak in the energy diagram around the radius of $200 \mathrm{~nm}$. This corresponds to the magnetic dipole resonance excitation, leading to strong confinement of optical energy inside the dielectric nanostructures [44]. There is a second peak at 300 $\mathrm{nm}$ corresponding to the toroidal dipole excitation. It shows that the toroidal dipole excitation in conjunction with a suppressed electric dipole can concentrate optical energy inside dielectric nanoparticles. Nevertheless, as mentioned earlier, other existing modes dominate the toroidal dipole excitation. Therefore, it is not surprising that the peak corresponding to the toroidal dipole excitation is not the strongest peak.

\section{B. Tightly focused radially polarized beam}

An alternative way to excite a toroidal dipole in the nanodisk is to use a tightly focused radially polarized beam that belongs to the family of cylindrical vector beams. This is a light source with an ultrasmall focal area and an inherently broken spatial-inversion symmetry. Toroidal excitation by this scheme is schematically shown in Fig. 3(a), where the dielectric nanodisk is placed at the focal point of a tightly focused radially polarized beam. When a beam with radial polarization illuminates a pupil, a solid longitudinal component of the electric field is created near the focal point. The azimuthal component of the electric field near the focus is zero, while the longitudinal $\left(E_{z}\right)$ and radial $\left(E_{\rho}\right)$ components are defined as follows [29]:

$$
\begin{aligned}
& E_{z}(\rho, z)=2 i A \int_{0}^{\alpha} \cos ^{\frac{1}{2}} \theta \sin ^{2} \theta l_{0}(\theta) J_{0}(k \rho \sin \theta) e^{i k z \cos \theta} d \theta, \\
& E_{\rho}(\rho, z)=A \int_{0}^{\alpha} \cos ^{\frac{1}{2}} \theta \sin (2 \theta) l_{0}(\theta) J_{1}(k \rho \sin \theta) e^{i k z \cos \theta} d \theta .
\end{aligned}
$$

We adopted a similar notation to that of Richards and Wolf [45] in which $\theta$ is the angle that a ray makes with the $z$ axis, and $\alpha$ is the angular aperture [see Fig. 3(a)], obtained by $\sin ^{-1}\left(\mathrm{NA} / n_{m}\right)$. We considered the numerical aperture NA $=$ 0.86 , and the medium refractive index $n_{m}=1$. The constant in the relation is $A=1$, the parameter $J$ refers to Bessel functions, and $l_{0}$ represents the relative amplitude of the field that is expressed as

$$
l_{0}(\theta)=\exp \left[-\beta_{0}^{2}\left(\frac{\sin \theta}{\sin \alpha}\right)^{2}\right] J_{1}\left(2 \beta_{0} \frac{\sin \theta}{\sin \alpha}\right) .
$$

The beam waist has been taken as $\beta_{0}=3 / 2$ in our calculation. A radially polarized beam has an azimuthal magnetic field component that can be considered analogous to the magnetic field distribution of a toroidal dipole.

Spherical scattering of the tightly focused radially polarized beam is illustrated in Fig. 3(b). It can be seen that electrical modes play the main role, while magnetic modes are suppressed. It demonstrates that the radial and longitudinal components of the electric field and the geometry of the disk have not induced the transverse current leading to the excitation of magnetic modes. Figure 3(c) shows the spherical electric dipole separated into the Cartesian electric and toroidal dipoles. For the radii larger than $300 \mathrm{~nm}$, the toroidal dipole is strongly excited, which is attributed to the existence of the longitudinal and in-plane electric fields of the incident light. Combining these two electric field components would lead to the drive of poloidal currents along the nanodisk's meridians and thus the excitation of the dipole toroid. Differences between the scattering characteristics of the tightly focused radially polarized beam and the plane wave [Figs. 2(b) and 2(c)] are observable. Unlike the plane wave case in which magnetic modes made significant contributions across the full radius range, a tightly focused radially polarized beam strongly suppressed magnetic modes. Furthermore, again unlike the case of the plane wave, Fig. 3(c) shows that the toroidal dipole becomes the dominant multipole up to quadrupole order for the radii larger than $300 \mathrm{~nm}$. Besides, there is another difference related to toroidal excitation. Three points corresponding to the electric dipole excitation, the toroidal dipole excitation, and the anapole state are marked in Fig. 3(b) and 3(c) by red, green, and blue circles, respectively. The tightly focused radially polarized light led to the toroidal dipole excitation at the radius of $355 \mathrm{~nm}$ (green circle) that suffers only from a weak EQ contribution, while for the plane wave case, off-resonant magnetic modes dominate the toroidal excitation and account for more than $70 \%$ of the total multipole scattering [see Figs. 2(b) and 2(c)].

Three-dimensional far-field radiation patterns corresponding to the three marked excitations are illustrated in Figs. 3(d)-3(f). The electric dipole and the toroidal dipole [the red and green circles in Fig. 3(c)] have almost identical radiation patterns with a slight difference due to the weak contribution of higher-order multipoles. Therefore, this nearideal toroidal dipole is not distinguishable from the electric dipole using the far-field radiation pattern. Cartesian multipolar decomposition analysis was required for its identification. Figure 3(f) shows the radiation pattern of the anapole state (the blue circle around $R=430 \mathrm{~nm}$ ). The dominant modes at this wavelength are the electric and toroidal dipoles, but these modes cancel most of the radiation of each other at the far field due to the formation of the anapole state. Therefore, 


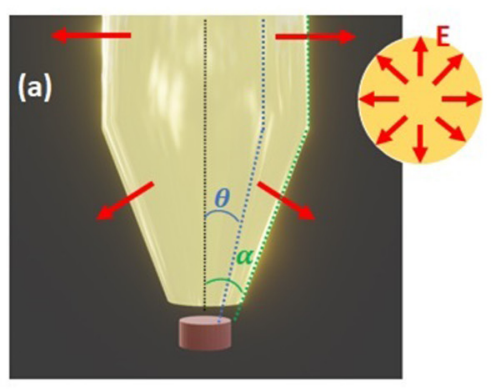

(d)

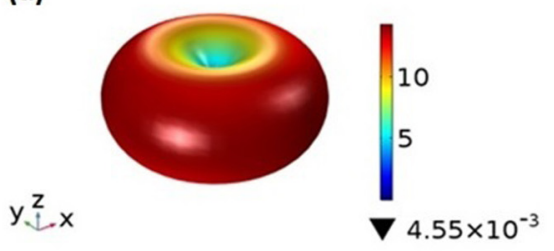

Electric dipole

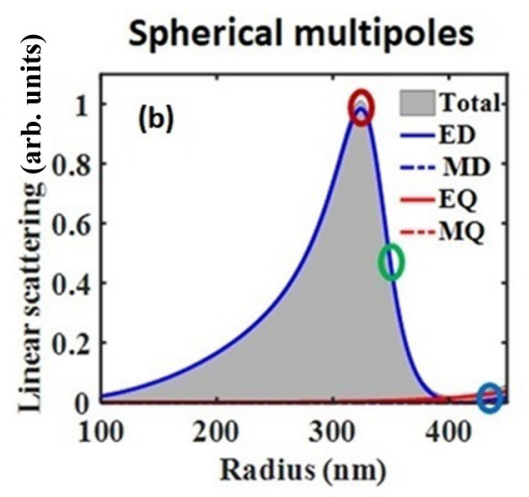

(e)

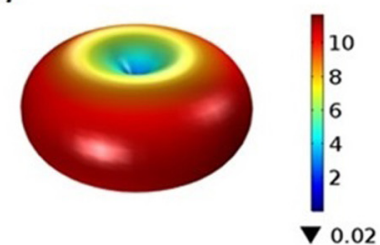

Toroidal dipole

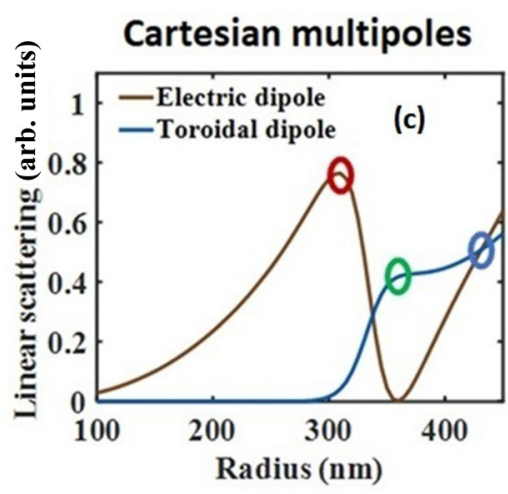

$\Delta 5$

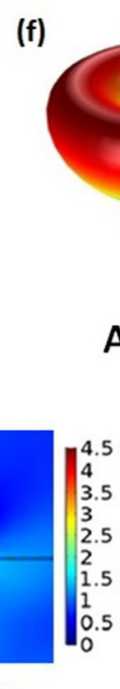

Toroidal dipole

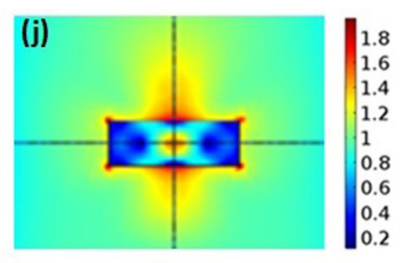

Anapole

FIG. 3. (a) Configuration of toroidal mode excitation by the tightly focused radially polarized beam. A tightly focused radially polarized beam in which both radial and longitudinal components of the electric field (red arrows) are built by a focusing lens illuminating a dielectric nanodisk. (b,c) Multipoles in the spherical and the Cartesian representations. Three points corresponding to the electric dipole excitation, the toroidal dipole excitation, and the anapole state are marked by red, green, and blue circles, respectively. (d-f) Far-field radiation patterns of three marked spots. (g) Electric field energy within the nanodisk as a function of radius. The maximum energy concentration within the nanodisk happens at toroidal excitation. ( $\mathrm{h}-\mathrm{j})$ Near-field distribution at three different excitations of the electric dipole, the toroidal dipole, and the anapole.

we can see far-field radiation that is somewhat similar to the typical quadrupole radiation pattern due to the presence of other modes, in particular the electric quadrupole.

We calculated the electric field energy within the nanodisk as a function of radius in Fig. $3(\mathrm{~g})$. There is a peak in the electric energy curve near the toroidal excitation. This arose from the confinement of the electromagnetic field inside the nanodisk due to toroidal dipole excitation. Thus, we achieved a significantly enhanced electric energy localized within the nanodisk. Unlike the plane wave excitation, there is no peak in the energy spectrum corresponding to magnetic excitation as the tightly focused radially polarized light has not significantly excited the magnetic modes. The near-field distribution of the electric field can confirm the electric energy localization inside the nanodisk. As shown in Figs. 3(h)-3(I), although both the electric dipole and the toroidal dipole show electric fields of the same intensity, the electric dipole excitation mainly distributes the electric field near the nanodisk. The toroidal excitation, in contrast, concentrates it inside the nan- odisk. For the anapole state, we can see electric energy is localized within the nanodisk although the spread of energy outside the nanodisk is also observable, which may come from the contribution of EQ and higher modes.

\section{Focused doughnut pulse}

Another solution to the homogeneous Maxwell's equations, known as pulse solution, refers to the localized electromagnetic energy propagating in the free space [46]. Of particular interest of the pulse solution is the focused doughnut pulse first introduced by Hellwarth and Nouchi [47]. The topology of electromagnetic fields in this kind of pulse is similar to toroidal multipoles in the matter, making it an interesting option to induce a toroidal response in nanophotonic systems. More recently, generation and observation of such toroidal light pulses have been reported by Zheludev's group [48]. Figure 4(a) schematically illustrates the nanodiskdoughnut pulse interaction. The focused doughnut pulse is a 


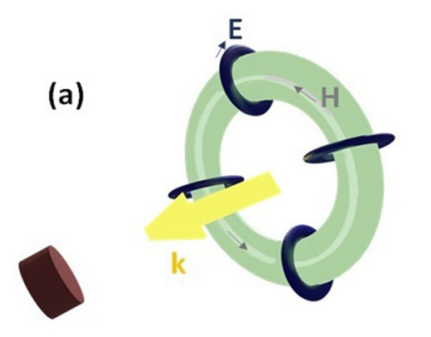

(b)
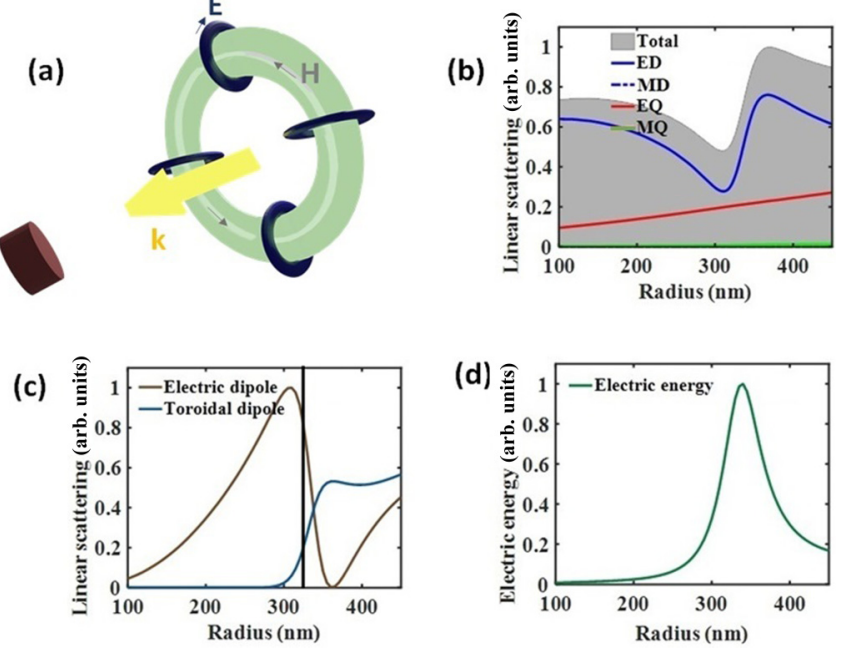

FIG. 4. (a) Configuration of toroidal mode excitation by a focused doughnut pulse. A transverse magnetic (TM) focused doughnut pulse illuminates a nanodisk at its focus point. Blue and gray arrows represent the $E$ and $H$ fields, respectively. (b) Multipolar decomposition in the spherical representation. A dip around $310 \mathrm{~nm}$ in the total scattering spectrum is observed that is attributed to an anapole state. (c) Multipolar decomposition in the Cartesian representation. Weak resonance around $360 \mathrm{~nm}$ corresponds to toroidal dipole excitation. Black line points to the nonideal anapole. (d) Electric energy within the nanodisk as a function of its radius.

single-cycle electromagnetic pulse in which fields are spatially localized within the toroidal configuration, converging to a focus and then diverging. The components of the fields of the transverse magnetic (TM) focused doughnut pulse are expressed in cylindrical coordinates $(\rho, \theta, z)$ as follows:

$$
\begin{aligned}
H_{\theta} & =4 i f_{0} \frac{\rho\left(q_{1}+q_{2}-2 i c t\right)}{\left[\rho^{2}+\left(q_{1}+i \tau\right)\left(q_{2}-i \sigma\right)\right]^{3}}, \\
E_{\rho} & =4 i f_{0} \sqrt{\frac{\mu_{0}}{\varepsilon_{0}}} \frac{\rho\left(q_{2}-q_{1}-2 i z\right)}{\left[\rho^{2}+\left(q_{1}+i \tau\right)\left(q_{2}-i \sigma\right)\right]^{3}}, \\
E_{z} & =-4 f_{0} \sqrt{\frac{\mu_{0}}{\varepsilon_{0}}} \frac{\rho^{2}-\left(q_{1}+i \tau\right)\left(q_{2}-i \sigma\right)}{\left[\rho^{2}+\left(q_{1}+i \tau\right)\left(q_{2}-i \sigma\right)\right]^{3}},
\end{aligned}
$$

where $\sigma=z+c t, \tau=z-c t, f_{0}$ is an arbitrary dimensionless constant for normalization, and the parameters $q_{1}$ and $q_{2}$ are, respectively, the wavelength of the doughnut pulse and the depth of the focal region [37,47]. The pulse with the parameter $q_{2}=100 q_{1}$ is considered and the nanodisk is located at $\rho=$ $z=0$. The pulse is simulated at the time $t=0$ corresponding to the focus point.

Here we study the interaction of the TM doughnut pulse with the nanodisk. Electric field components (longitudinal and radial) are an analog to the poloidal currents, and the azimuthal magnetic field is analogous to the closed loop of the $\underline{H}$ field. Due to the toroidal configuration of the doughnut pulse, we expect the toroidal mode excitation inside the nanodisk. We first analyzed the multipoles excited inside the nanodisk up to quadrupole to uncover the nanodisk-doughnut pulse interaction characteristics. Contributions of the multipoles to the scattering for various radii nanodisks are illustrated in
Figs. 4(b) and 4(c). For smaller particles, like the focused radially polarized case, the electric dipole is dominant because of the strong coupling between the nanodisk and the longitudinal component of the electric field in Eq. (11). This dominant mode is, however, accompanied by an electric quadrupole contribution. As both nanodisk and the focused doughnut pulse have cylindrical symmetry, it is not surprising that the magnetic response in Fig. 4(b) is suppressed over the radius range of interest. As opposed to magnetic modes, the electric quadrupole exists over the full radius range, possibly due to the varying radial electric field across the nanodisk. When the radius increased beyond $300 \mathrm{~nm}$, the toroidal dipole became the dominant mode up to quadrupole order, as can be noticed from Fig. 4(c). It is evidence of the poloidal currents flowing along the meridians of the disk produced by the interplay of both longitudinal and radial electric fields. Furthermore, toroidal dipole excitation around $360 \mathrm{~nm}$ shows a weak resonance. This is an exciting feature because it indicates that the focused doughnut pulse is a promising option for exciting a resonant toroidal response accompanied by a vanished electric dipole in metamaterials with nontoroidal configurations. We also observe a dip around $310 \mathrm{~nm}$ in the total scattering spectrum in Fig. 4(b) that is attributed to an anapole state where a suppressed scattering is observed despite the presence of both electric and toroidal dipoles. However, this anapole state is not pure, and multipolar analysis is more complex. First of all, the electric and toroidal moments do not possess an exactly equal magnitude [black line in Fig. 4(c)]; therefore, a part of the far-field radiation remains. As a result, the spherical ED magnitude at the anapole state [the dip in Fig. 4(b)] did not become zero. Furthermore, the contribution of the electric quadrupole is not negligible, and its effect on the anapole cannot be ignored. Moreover, we considered a limited number of modes (up to the second order of the expansion) in the multipolar analysis. Thus there might be higher multipoles affecting this state. Overall, a pure and perfect anapole excitation cannot be obtained by a free space electromagnetic source [27]. Despite all that, we can still see the confinement of electric energy at the anapole and toroidal resonance. Figure 4(d) shows electric energy within the particle as a function of its radius. The energy curve exhibits a peak, but it is neither at the anapole nor at the toroidal resonance due to the complexity of multipoles' contribution, as discussed earlier. However, it is located near the anapole and toroidal resonance which confirms the confinement of electric energy inside the nanodisk at these two states. In fact, both anapole state and toroidal resonance exhibit an energy concentration of about $65 \%$.

\section{CONCLUSION}

We studied toroidal dipole excitation in an individual dielectric nanodisk under structured light illumination. Two structured lights have been investigated: the tightly focused radially polarized light and the focused doughnut pulse. Such structured lights can strongly interact with dielectric nanostructures due to the versatile polarization and spatial field distribution. Our results suggest that structured lights are more powerful tools for the resonant excitation of toroidal modes in nontoroidal structures than plane wave illumi- 
nation. They can significantly suppress the contribution of magnetic multipoles and allow the toroidal dipole to be the leading term in the optical response of nanodisks. The tightly focused radially polarized beam excited a near-ideal toroidal dipole moment with a far-field radiation pattern similar to an electric dipole moment. Furthermore, it was shown that the toroidal-driven field confinement leads to an energy concentration within the nanodisk. Therefore, the structured light excitation of the toroidal response could potentially enhance the linear and nonlinear light-matter interactions and tailor subwavelength absorbers in all-dielectric nanophotonics.
[1] G. Mie, Contributions to the optics of turbid media, particularly of colloidal metal solutions, Ann. Phys. 25, 377 (1908).

[2] Y. S. Kivshar, W. L. Barnes, O. Hess, and N. Zheludev, New horizons for nanophotonics subject areas, Philos. Trans. R. Soc., A 375, 20160380 (2017).

[3] Y. Kivshar and A. E. Miroshnichenko, Meta-optics with Mie resonances, Opt. Photonics News 28, 24 (2017).

[4] V. M. Dubovik and V. V. Tugushev, Toroid moments in electrodynamics and solid-state physics, Phys. Rep. 187, 145 (1990).

[5] N. Papasimakis, V. A. Fedotov, V. Savinov, T. A. Raybould, and N. I. Zheludev, Electromagnetic toroidal excitations in matter and free space, Nat. Mater. 15, 263 (2016).

[6] I. B. Zel'Dovich, Electromagnetic interaction with parity violation, Sov. J. Exp. Theor. Phys. 6, 1184 (1958).

[7] J. Li, X.-X. Xin, J. Shao, Y.-H. Wang, J.-Q. Li, L. Zhou, and Z.-G. Dong, From non- to super-radiating manipulation of a dipolar emitter coupled to a toroidal metastructure, Opt. Express 23, 29384 (2015).

[8] E. A. Gurvitz, K. S. Ladutenko, P. A. Dergachev, A. B. Evlyukhin, A. E. Miroshnichenko, and A. S. Shalin, The highorder toroidal moments and anapole states in all-dielectric photonics, Laser Photonics Rev. 13, 1800266 (2019).

[9] B. Luk'yanchuk, R. Paniagua-Domínguez, A. I. Kuznetsov, A. E. Miroshnichenko, and Y. S. Kivshar, Hybrid anapole modes of high-index dielectric nanoparticles, Phys. Rev. A 95, 063820(R) (2017).

[10] L. Hüttenhofer, F. Eckmann, A. Lauri, J. Cambiasso, E. Pensa, Y. Li, E. Cortés, I. D. Sharp, and S. A. Maier, Anapole excitations in oxygen-vacancy-rich $\mathrm{TiO}_{2-x}$ nanoresonators: Tuning the absorption for photocatalysis in the visible spectrum, ACS Nano 14, 2456 (2020).

[11] N. Zhou, V. López-Puente, Q. Wang, L. Polavarapu, I. Pastoriza-Santos, and Q.-H. Xu, Plasmon-enhanced light harvesting: Applications in enhanced photocatalysis, photodynamic therapy and photovoltaics, RSC Adv. 5, 29076 (2015).

[12] W. Hu, H. Cong, W. Huang, Y. Huang, L. Chen, A. Pan, and C. Xue, Germanium/perovskite heterostructure for highperformance and broadband photodetector from visible to infrared telecommunication band, Light Sci. Appl. 8, 106 (2019).

[13] O. Mitrofanov, L. L. Hale, P. P. Vabishchevich, T. S. Luk, S. J. Addamane, J. L. Reno, and I. Brener, Perfectly absorbing dielectric metasurfaces for photodetection, APL Photonics $\mathbf{5}$, 101304 (2020).

[14] R. Maiti, C. Patil, M. A. S. R. Saadi, T. Xie, J. G. Azadani, B. Uluutku, R. Amin, A. F. Briggs, M. Miscuglio, D. Van Thourhout, S. D. Solares, T. Low, R. Agarwal, S. R. Bank, and V. J. Sorger, Strain-engineered high-responsivity $\mathrm{MoTe}_{2}$ photodetector for silicon photonic integrated circuits, Nat. Photonics 14, 578 (2020).
[15] Z. Yu, X. Xi, J. Ma, H. K. Tsang, C.-L. Zou, and X. Sun, Photonic integrated circuits with bound states in the continuum, Optica 6, 1342 (2019).

[16] H. Hasebe, H. Sugimoto, T. Hinamoto, and M. Fujii, Coupled toroidal dipole modes in silicon nanodisk metasurface: Polarization independent narrow band absorption and directional emission, Adv. Opt. Mater. 8, 2001148 (2020).

[17] C. Rosales-Guzmán, B. Ndagano, and A. Forbes, A review of complex vector light fields and their applications, J. Opt. 20, 123001 (2018).

[18] C. Xu, H. Hu, Y. Liu, and D. Deng, Radially polarized symmetric Airy beam Opt. Lett. 45, 1451 (2020).

[19] F. Xiao, J. Zhang, W. Yu, W. Zhu, T. Mei, M. Premaratne, and J. Zhao, Reversible optical binding force in a plasmonic heterodimer under radially polarized beam illumination, Opt. Express 28, 3000 (2020).

[20] S. W. Jolly, On the importance of frequency-dependent beam parameters for vacuum acceleration with few-cycle radially polarized laser beams, Opt. Lett. 45, 3865 (2020).

[21] F. Feng, G. Si, C. Min, X. Yuan, and M. Somekh, On-chip plasmonic spin-Hall nanograting for simultaneously detecting phase and polarization singularities, Light Sci. Appl. 9, 95 (2020).

[22] S. Reich, N. S. Mueller, and M. Bubula, Selection rules for structured light in nanooligomers and other nanosystems, ACS Photonics 7, 1537 (2020).

[23] J. Sancho-Parramon and S. Bosch, Dark modes and Fano resonances in plasmonic clusters excited by cylindrical vector beams, ACS Nano 6, 8415 (2012).

[24] Y. Shen, X. Wang, Z. Xie, C. Min, X. Fu, Q. Liu, M. Gong, and $\mathrm{X}$. Yuan, Optical vortices 30 years on: OAM manipulation from topological charge to multiple singularities, Light Sci. Appl. 8, 90 (2019).

[25] A. Forbes, Structured light: Tailored for purpose, Opt. Photonics News 31, 24 (2020).

[26] H. Larocque, A. D’Errico, M. F. Ferrer-Garcia, A. Carmi, E. Cohen, and E. Karimi, Optical framed knots as information carriers, Nat. Commun. 11, 5119 (2020).

[27] T. Raybould, V. A. Fedotov, N. Papasimakis, I. Youngs, and N. I. Zheludev, Exciting dynamic anapoles with electromagnetic doughnut pulses, Appl. Phys. Lett. 111, 081104 (2017).

[28] L. Wei, Z. Xi, N. Bhattacharya, and H. P. Urbach, Excitation of the radiationless anapole mode, Optica 3, 799 (2016).

[29] K. S. Youngworth and T. G. Brown, Focusing of high numerical aperture cylindrical-vector beams, Opt. Express 7, 77 (2000).

[30] A. Zdagkas, N. Papasimakis, V. Savinov, M. R. Dennis, and N. I. Zheludev, Singularities in the flying electromagnetic doughnuts, Nanophotonics 8, 1379 (2019).

[31] L. Xu, M. Rahmani, K. Zangeneh Kamali, A. Lamprianidis, L. Ghirardini, J. Sautter, R. Camacho-Morales, H. Chen, M. Parry, 
I. Staude, G. Zhang, D. Neshev, and A. E. Miroshnichenko, Boosting third-harmonic generation by a mirror-enhanced anapole resonator, Light Sci. Appl. 7, 44 (2018).

[32] A. E. Miroshnichenko, A. B. Evlyukhin, Y. F. Yu, R. M. Bakker, A. Chipouline, A. I. Kuznetsov, B. Luk'yanchuk, B. N. Chichkov, and Y. S. Kivshar, Nonradiating anapole modes in dielectric nanoparticles, Nat. Commun. 6, 8069 (2015).

[33] K. Koshelev, G. Favraud, A. Bogdanov, Y. Kivshar, and A. Fratalocchi, Nonradiating photonics with resonant dielectric nanostructures, Nanophotonics 8, 725 (2019).

[34] M. Gupta and R. Singh, Toroidal metasurfaces in a 2D flatland, Rev. Phys. 5, 100040 (2020).

[35] R. E. Noskov, I. I. Shishkin, H. Barhom, and P. Ginzburg, NonMie optical resonances in anisotropic biomineral nanoparticles, Nanoscale 10, 21031 (2018).

[36] A. Karabchevsky, On-chip optical vortex-based nanophotonic detectors, Light Sci. Appl. 9, 115 (2020).

[37] T. Raybould, V. Fedotov, N. Papasimakis, I. Youngs, and N. Zheludev, Focused electromagnetic doughnut pulses and their interaction with interfaces and nanostructures, Opt. Express 24, 3150 (2016).

[38] Y. Bao, X. Zhu, and Z. Fang, Plasmonic toroidal dipolar response under radially polarized excitation, Sci. Rep. 5, 11793 (2015).

[39] J. A. Parker, H. Sugimoto, B. Coe, D. Eggena, M. Fujii, N. F. Scherer, S. K. Gray, and U. Manna, Excitation of Nonradiating Anapoles in Dielectric Nanospheres, Phys. Rev. Lett. 124, 097402 (2020).
[40] D. J. Griffiths, Introduction to Electrodynamics, 3rd ed. (Prentice-Hall, Inc., Hoboken, NJ, 1999).

[41] N. Talebi, S. Guo, and P. A. Van Aken, Theory and applications of toroidal moments in electrodynamics: Their emergence, characteristics, and technological relevance, Nanophotonics 7, 93 (2018).

[42] P. Grahn, A. Shevchenko, and M. Kaivola, Electromagnetic multipole theory for optical nanomaterials, New J. Phys. 14, 093033 (2012).

[43] Z.-G. Dong, J. Zhu, J. Rho, J.-Q. Li, C. Lu, X. Yin, and $\mathrm{X}$. Zhang, Optical toroidal dipolar response by an asymmetric double-bar metamaterial, Appl. Phys. Lett. 101, 144105 (2012).

[44] P. D. Terekhov, K. V. Baryshnikova, Y. Greenberg, Y. H. Fu, A. B. Evlyukhin, A. S. Shalin, and A. Karabchevsky, Enhanced absorption in all-dielectric metasurfaces due to magnetic dipole excitation, Sci. Rep. 9, 1 (2019).

[45] B. Richards and E. Wolf, Electromagnetic diffraction in optical systems. II. Structure of the image field in an aplanatic system, Proc. R. Soc. A 253, 358 (1959).

[46] J. N. Brittingham, Focus waves modes in homogeneous Maxwell's equations: Transverse electric mode, J. Appl. Phys. 54, 1179 (1983).

[47] R. W. Hellwarth and P. Nouchi, Focused one-cycle electromagnetic pulses, Phys. Rev. E 54, 889 (1996).

[48] A. Zdagkas, Y. Shen, C. McDonnell, J. Deng, G. Li, T. Ellenbogen, N. Papasimakis, and N. I. Zheludev, Observation of toroidal pulses of light, arXiv:2102.03636. 